\title{
Numerical estimation of critical parameters using the bond entropy
}

\author{
Rafael A. Molina ${ }^{1}$ and Peter Schmitteckert ${ }^{2}$ \\ ${ }^{1}$ Instituto de Estructura de la Materia-CSIC, Serrano 123, 28006 Madrid, Spain \\ ${ }^{2}$ Institut für Theorie der Kondensierten Materie, Universität Karlsruhe, 76128 Karlsruhe, Germany
}

(Received 3 January 2007; revised manuscript received 2 March 2007; published 6 June 2007)

\begin{abstract}
Using a model of spinless fermions in a lattice with nearest-neighbor and next-nearest-neighbor interactions, we show that the entropy of the reduced two-site density matrix (the bond entropy) can be used as an extremely accurate and easy to calculate numerical indicator for the critical parameters of the quantum phase transition when the basic ordering pattern has a two-site periodicity. The actual behavior of the bond entropy depends on the particular characteristics of the transition under study. For the Kosterlitz-Thouless-type phase transition from a Luttinger liquid phase to a charge-density wave state, the bond entropy has a local maximum, while in the transition from the Luttinger liquid to the phase separated state, the derivative of the bond entropy has a divergence due to the cancellation of the third eigenvalue of the two-site reduced density matrix.
\end{abstract}

DOI: 10.1103/PhysRevB.75.235104

PACS number(s): 73.43.Nq, 03.67.Mn, 75.10.Jm

\section{INTRODUCTION}

A quantum phase transition (QPT) is a qualitative change in the ground state of a quantum system as some parameter is varied. ${ }^{1,2}$ Contrary to classical phase transitions, QPTs occur at zero temperature and are due to the effect of quantum fluctuations and not of thermal fluctuations. The previous definition is very general; however, the abrupt change in the structure of the ground state that defines the phase transition can have different consequences depending on the different cases. The ground-state energy may become nonanalytic when approaching the critical parameter. The energy gap between the ground state and the first excited state may go to zero in the critical point. The correlations at the critical point may decay as power laws instead of exponentially, indicating a diverging correlation length. It is possible to find quantum systems which have some of these indications of the QPT and not others. ${ }^{3,4}$ For this reason, alternative ways for the classification of QPTs and for the numerical investigation of the critical parameters in a QPT can be very helpful.

In recent years, quantum information concepts have started to be applied to the study of QPTs. One central concept in quantum information theory is the concept of entanglement. ${ }^{5}$ Two quantum systems in a pure state are entangled if their state cannot be written as the product of two separate pure states for each of the quantum systems. Entanglement measures quantum correlations, and as correlations are typically maximal at the critical points of QPTs, it was realized that some entanglement measures may have a singularity or a maximum at the critical point. The amount of entanglement has been shown to be a very sensitive quantity to the value of the critical parameter governing the phase transition. ${ }^{6-10}$ In particular, concurrence ${ }^{11}$ has been used to investigate spin models, and this quantity shows an extreme or singular behavior at the corresponding critical points. ${ }^{7}$ The block-block entanglement between two parts of the system has also been used, establishing connections with conformal field theory. $8,12,13$

In a recent work, $\mathrm{Gu}$ et al. analyzed the local entanglement and its relationship with phase transitions in the onedimensional and two-dimensional XXZ spin models. ${ }^{14}$ The local entanglement was measured with the von Neumann entropy of the two-site density matrix. It is more convenient numerically than the block-block entanglement as the size of the density matrix needed for the latter quantity grows exponentially with the size of the block. Using Bethe ansatz results for the one-dimensional $X X Z$ model, Gu et al. showed that the local information obtained from the entanglement entropy of the two-site density matrix is enough to study the phase transitions that occur in this model. Considering blocks larger than the characteristic length scales of the system (that in the critical point diverge) was shown to be unnecessary as the entanglement between a block of two spins and the rest of the system is sufficient to reveal the most important information about the system. Their results hint to the possibility of using these properties for the numerical study of phase transitions with small systems.

It is the purpose of this work to study numerically the local entanglement as a function of the size of the system. In particular, we will concentrate in the critical points of the phase transitions. We will study a one-dimensional model of spinless fermions with nearest-neighbor interactions that can be transformed through the Jordan-Wigner transformation to the $X X Z$ model with spins at each site $S_{j}=1 / 2 .{ }^{15}$ For repulsive interaction $V_{1}=2.0$, the ground state performs a Kosterlitz-Thouless (KT)-type phase transition from a Luttinger liquid to a charge-density wave, which corresponds to an antiferromagnetically ordered state in the spin picture. For attractive interaction $V_{1}=-2.0$, there is a phase transition to a phase separated state, which corresponds to a ferromagnet in the spin picture. We will show that the bond entropy $S_{\text {bond }}$ enables us to determine the critical points of both quantum phase transitions with an astonishing accuracy, albeit they present very different characteristics and symmetries. We will show how these differences are reflected in the behavior of the bond entropy. In the model we use, the different matrix elements of the two-site density matrix can be written easily as a function of different two-site correlation functions as we show in Sec. II. These relations are the basis of the sensitivity of the bond entropy to QPT. Similar formulas should be found for different models, although the particular details can be different. The advantage of the bond entropy over the direct determination of the correlation functions is 
the behavior of the finite-size correlations. These finite-size correlations are specially important for the KT transition. Calculations based on DMRG have failed to address effectively the question of the determination of the critical parameters in this case, although some improvement has been reached recently. ${ }^{16}$ We can say that the bond entropy is a special correlation function with particular ease in treating finite-size effects. In Sec. III, we will show that the bond entropy has a maximum at the critical point of the KT transition for repulsive interactions, and we will calculate the size dependence of the curvature of the bond entropy at this maximum. The QFT to the phase separated state for attractive interactions will be studied in Sec. IV. In this case, one of the eigenvalues of the two-site density matrix goes to zero at the critical point and the bond entropy has a singularity. In Sec. V, we include next-nearest-neighbor interaction to test the generality of our finding. The effect of longer ranged interaction was studied before in the context of multiple Umklapp scattering. ${ }^{16}$ In this case, the interaction can now lead to phase transitions at fillings different from $1 / 2$ and to ordering patterns with increased unit cell. Finally, in Sec. VI, we will present some conclusions.

\section{BOND ENTROPY FOR THE NEAREST-NEIGHBOR INTERACTION MODEL}

We shall consider the one-dimensional spinless fermions model with next-neighbor interactions,

$$
\hat{H}=-t \sum_{i=1}^{L}\left(\hat{c}_{i}^{\dagger} \hat{c}_{i-1}+\hat{c}_{i-1}^{\dagger} \hat{c}_{i}\right)+\sum_{i=1}^{L} V_{1}\left(\hat{n}_{i}-\frac{1}{2}\right)\left(\hat{n}_{i-1}-\frac{1}{2}\right),
$$

where the operators appearing in the formula are the usual fermionic creation, annihilation, and number operators at site $i, L$ is the total number of sites, $t$ is the hopping matrix element between neighboring sites, and $V_{1}$ is the nearestneighbor interaction strength. An important property of this model is that it can be transformed into the $X X Z$ spin $S=1 / 2$ model through the Jordan-Wigner transformation, ${ }^{15,17}$

$$
\begin{gathered}
\hat{S}_{j}^{-}=\exp \left(-i \pi \sum_{\ell=1}^{j-1} \hat{c}_{\ell}^{\dagger} \hat{c}_{\ell}\right) c_{j}, \\
\hat{S}_{j}^{z}=\hat{n}_{j}-1 / 2 .
\end{gathered}
$$

For an even number of particles, a phase term appears in the boundary condition when we apply the Jordan-Wigner transformation to Hamiltonian [Eq. (1)]. As we are not interested in this even-odd effect, we will consider periodic (antiperiodic) boundary conditions $c_{0} \equiv c_{M}\left(c_{0} \equiv-c_{M}\right)$ for $N$ odd (even), and both models will be equivalent in our examples. Although we will mainly consider the spinless fermion model, we will make comments regarding the equivalent behavior of both models when we believe it will be useful to clarify some situation (specially in the "ferromagnetic" phase). The Hamiltonian [Eq. (1)] commutes with the total $\hat{N}=\sum \hat{n}_{i}$ operator, so the total number of particles is a con-

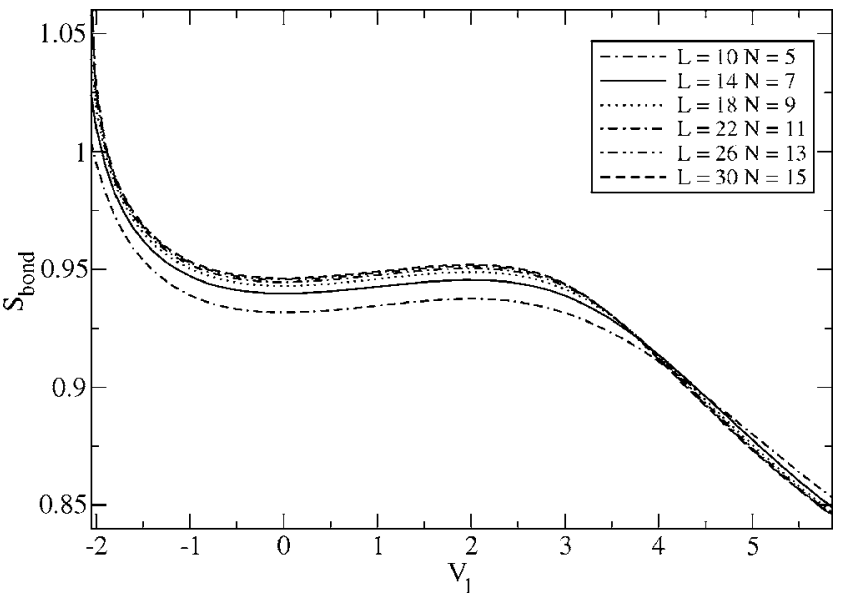

FIG. 1. Bond entropy $S_{\text {bond }}$ as a function of the interaction for different sizes of the spinless fermions ring $L$ at half filling $N$ $=L / 2$. The results for $L=26$ and $L=30$ are hardly distinguishable. We have a maximum of $S_{\text {bond }}$ at $V_{1}=2$ with extremely good approximation, see next figure, that marks the CDW insulator transition. The slope of the entropy diverges at $V_{1}=-2$, marking the appearance of the ferromagnetic transition.

served quantity and we will considering subspaces with a definite number of fermions, equivalent to consider subspaces with a definite value of $S_{z}$ in the $X X Z$ model.

This model has an interesting phase diagram depending on the value of the ratio of the interaction parameter and the hopping term $V_{1} / t$ and also on the number of particles $N$. Without loss of generality, we can consider $t=1$ and consider the phases as we change $V_{1}$. For $V_{1}<-2$, the equivalent spin system is ferromagnetic and the ground state is fully spin polarized. When we cross the first transition point $V_{c a}=-2$, the ground state of the system can be shown to be nondegenerate and with spin $S=0 .{ }^{18}$ Only in the half-filled case, $N$ $=L / 2$, there is another transition point at $V_{c b}=2$. For $V_{1}>2$, the system is a charge-density-wave-type insulator, the transition is of the Kosterlitz-Thouless type, and the order parameter depends exponentially on the difference $V-V_{c b}$, making an accurate numerical determination of the transition point notoriously hard.

We will define the bond entropy as the von Neumann entropy of the reduced density matrix of two-neighboring sites $\hat{\rho}_{i i+1}$. As a result of the conservation of $N$, the reduced density matrix can be written as a $4 \times 4$ matrix with three sectors of $N=0, N=1$, and $N=2$. In the two-site basis $|00\rangle$, $|01\rangle,|10\rangle$, and $|11\rangle$, it can be represented as

$$
\hat{\rho}_{i i+1}=\left(\begin{array}{cccc}
u^{-} & 0 & 0 & 0 \\
0 & \omega & z & 0 \\
0 & z^{*} & \omega & 0 \\
0 & 0 & 0 & u^{+}
\end{array}\right) .
$$

For this particular model using its invariance under translations, it can be shown that we can write these matrix elements in terms of certain correlation functions, ${ }^{19,20}$

$$
u^{-}=1+\left\langle\hat{n}_{i} \hat{n}_{i+1}\right\rangle-\frac{3}{2}\left\langle\hat{n}_{i}\right\rangle-\frac{1}{2}\left\langle\hat{n}_{i+1}\right\rangle,
$$




$$
\begin{gathered}
u^{+}=\left\langle\hat{n}_{i} \hat{n}_{i+1}\right\rangle+\frac{1}{2}\left\langle\hat{n}_{i}\right\rangle-\frac{1}{2}\left\langle\hat{n}_{i+1}\right\rangle, \\
\omega=\frac{1}{2}\left\langle\hat{n}_{i}\right\rangle+\frac{1}{2}\left\langle\hat{n}_{i+1}\right\rangle-\left\langle\hat{n}_{i} \hat{n}_{i+1}\right\rangle, \\
z=\frac{1}{2}\left(\left\langle\hat{c}_{i}^{\dagger} \hat{c}_{i+1}\right\rangle+\left\langle\hat{c}_{i+1}^{\dagger} \hat{c}_{i}\right\rangle\right) .
\end{gathered}
$$

We define the bond entropy $S_{\text {bond }}^{i}$ as the von Neumann entropy of the two-site density matrix $\hat{\rho}_{i i+1}$. In the model under study, the invariance under translations also implies that $S_{\text {bond }}^{i}$ does not depend on the site $i$,

$$
S_{\text {bond }}=-\sum_{j=1}^{4} \lambda_{j} \ln \lambda_{j},
$$

where $\lambda_{j}$ are the four eigenvalues of the reduced two-site density matrix $\hat{\rho}_{i i+1}$.

\section{NUMERICAL RESULTS}

In this section, we will show the numerical results for the bond entropy as a function of the size of the system using the DMRG algorithm. ${ }^{21}$

In Fig. 1, we show the results of $S_{\text {bond }}$ at half filling for different number of sites $L$. The behavior of the bond entropy around the two critical points is very different, reflecting the different changes in the symmetries and correlations of the ground state. The slope of $S_{\text {bond }}$ diverges around $V_{c a}=-2$ (we will explain that in more detail in the next section), while $S_{\text {bond }}$ is continuous but has a local maximum in the proximity of the second critical point $V_{c b}=2$. In both cases, one can understand the behavior of $S_{\text {bond }}$ from the behavior of the correlation functions in the different phases. ${ }^{14}$ More importantly, one can estimate with extraordinary precision the value of the critical parameter from very small system sizes even in the case of the transition to the charge-density wave (CDW) phase. In addition, $S_{\text {bond }}$ has a minimum for $V_{1}=0$.

In the top panel of Fig. 2, we see a zoom of the Fig. 1 for one particular case with parameter $L=30, N=15$ in the region around $V_{1}=2$. When we numerically calculate the position of the maximum with DMRG, we obtain the value of $V_{1}$ with a precision better than $10^{-5}$. Of course, in order to take full advantage of these properties of $S_{\text {bond }}$, we need a very accurate algorithm such as DMRG. We used at least 500 states per block for the $L \leqslant 30$ sites, leading to a discarded entropy below $10^{-9}$, and 1400 states per block for the 36 site system (see below), including the five lowest-lying states in order to treat the degeneracies correctly, leading to a discarded entropy typically below $10^{-10}$ and up to $2 \times 10^{-8}$ close to the phase transitions and always applied 11 finite lattice sweeps. Close to the CDW-I-Luttinger-liquid transition (see below), we used at least 2050 states per block and only two low-lying states to check our results. This resulted in a discarded entropy below $10^{-12}$. We would like to note that despite the large number of states, the DMRG runs are much cheaper as compared to the calculations in Ref. 16,

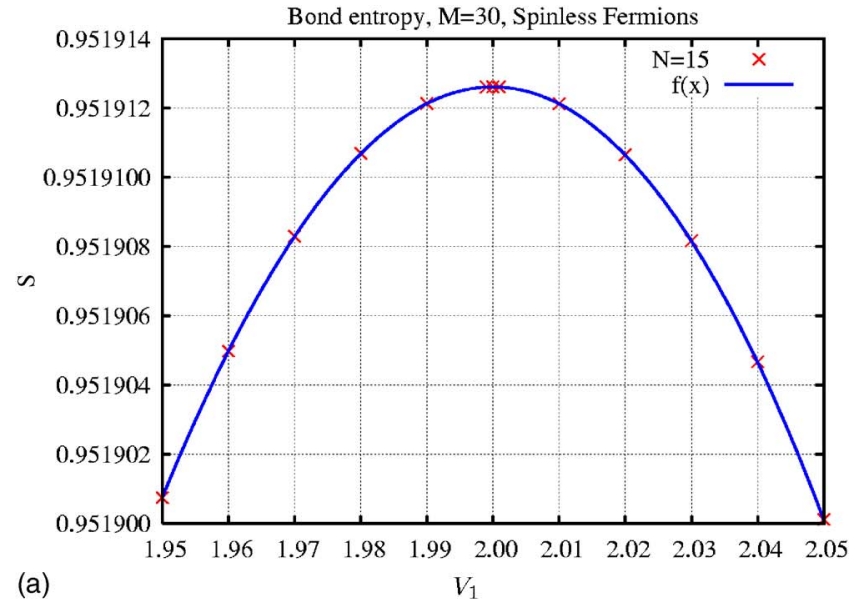

(a)

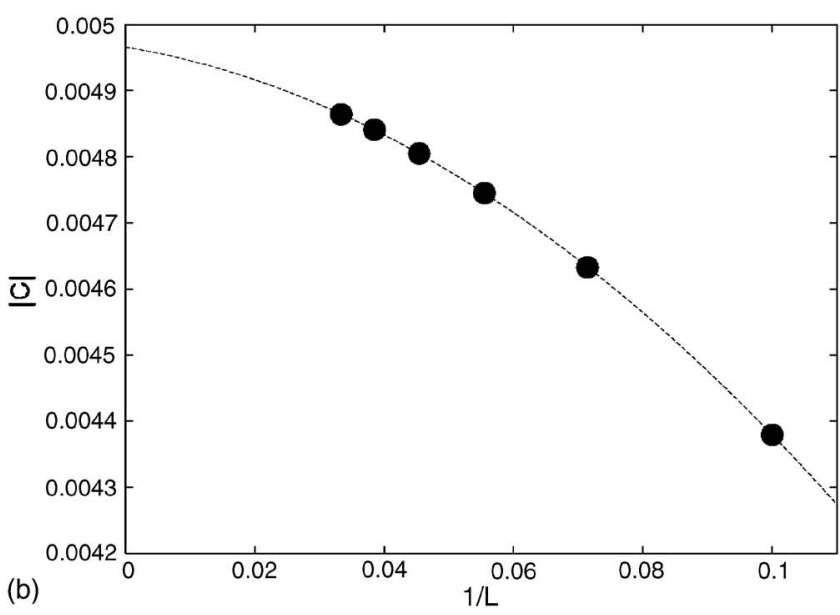

FIG. 2. (Color online) Top panel: Behavior of $S_{\text {bond }}$ as a function of $V_{1}$ in the neighborhood of the phase transition at $V_{1}=2$ in the case $L=30, N=15$. We can numerically pinpoint the maximum of the curve at $V_{1}=2$ with a precision better than $10^{-5}$. The function $f(x)$ is the second-order polynomial fit used to obtain the maximum of the curve. Bottom panel: Size dependence of the curvature of the bond entropy at the maximum.

since no resolvent has to be computed, e.g., the largest run took about a hundred CPU minutes. In the bottom panel, we show the size dependence of the curvature of the bond entropy near the maximum (at the critical point). The curvature increases with size so the maximum is more pronounced. The size scaling of the actual value of $V_{1}$ is not very significant as all points are very close to the critical value and within the error bars of the fit.

In Fig. 3, we see some examples comparing results at half filling and outside half filling. The qualitative behavior of the bond entropy is exactly the same around the first critical point $V_{c a}=-2$, but the maximum of the bond entropy around $V_{c b}=2$ disappears as soon as we move outside half filling, reflecting the absence of the $\mathrm{CDW}$ transition for fillings different from $1 / 2$.

\section{FERROMAGNETISM AND THE TWO-SITE DENSITY MATRIX}

As we have mentioned before, the slope of $S_{\text {bond }}$ diverges at $V_{1}=V_{c a}$. In Fig. 4, we show the results for the value of the 


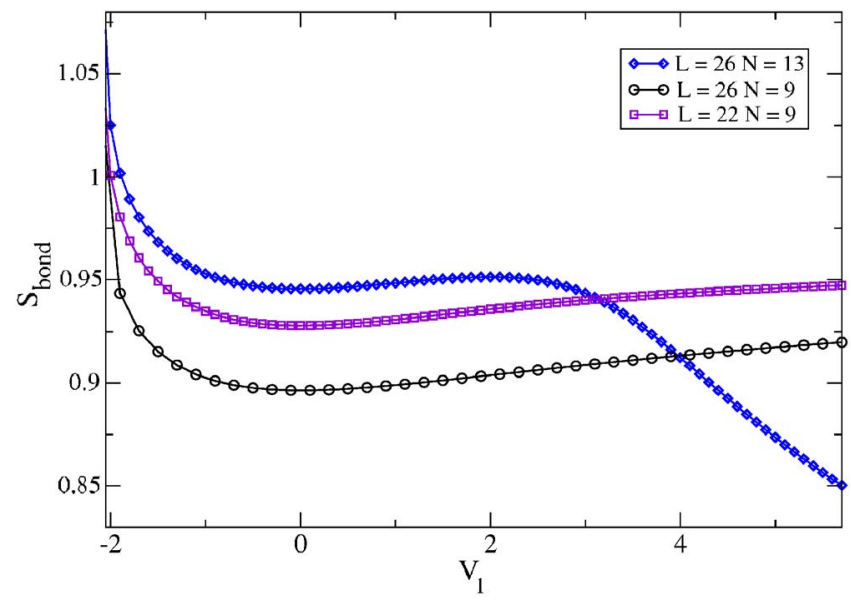

FIG. 3. (Color online) Behavior of $S_{\text {bond }}$ as a function of $V$ for $L=26, N=13$ (half filling) and $L=26, N=9$ and $L=22, N=9$ (outside half filling). We observe the same behavior in the ferromagnetic transition around $V_{c a}=-2$ but a complete different one in the transition around $V_{c b}=2$.

third eigenvalue of the two-site density matrix $\lambda_{3}$ for different sizes. We can reach a very high numerical precision in the determination of the ferromagnetic critical point studying the cancellation of the third eigenvalue of the two-site density matrix.

In the thermodynamic limit at $V_{1}=-2, \omega=z=1 / 4$ in Eq. (4). One can immediately see that the equality of both matrix elements implies that one of the eigenvalues of the density matrix is zero, which leads to the singularity of $S_{\text {bond }}$. If one tries a direct numerical examination of the values of the correlation functions independently, one does not get a very accurate estimation of the critical parameter. However, the examination of the particular combination appearing in the von Neumann entropy of the two-site density matrix allows a very accurate calculation even with very small system sizes due to the fact that although of $\omega$ and $z$ converge slowly to the thermodynamic limit value of $1 / 4$, their difference converges very quickly to zero at the critical value of the interaction.

\section{NEXT-NEAREST-NEIGHBOR INTERACTION MODEL}

In order to test the generality of our conclusions and to obtain a critical parameter of a phase transition in a model not solvable with Bethe ansatz, we add next-nearestneighbor interaction,

$$
\begin{aligned}
\hat{H}= & -t \sum_{i=1}^{L}\left(\hat{c}_{i}^{\dagger} \hat{c}_{i-1}+\hat{c}_{i-1}^{\dagger} \hat{c}_{i}\right)+\sum_{i=1}^{L} V\left(\hat{n}_{i}-\frac{1}{2}\right)\left(\hat{n}_{i-1}-\frac{1}{2}\right) \\
& +\sum_{i=1}^{L} V_{2}\left(\hat{n}_{i}-\frac{1}{2}\right)\left(\hat{n}_{i-2}-\frac{1}{2}\right),
\end{aligned}
$$

where $V_{2}$ is the strength of the interaction between sites separated by two lattice spacings. This Hamiltonian has been used to study the physics of materials that exhibit multiple phase transitions. Usually, one considers $V_{2}<V_{1}$ as one ex-

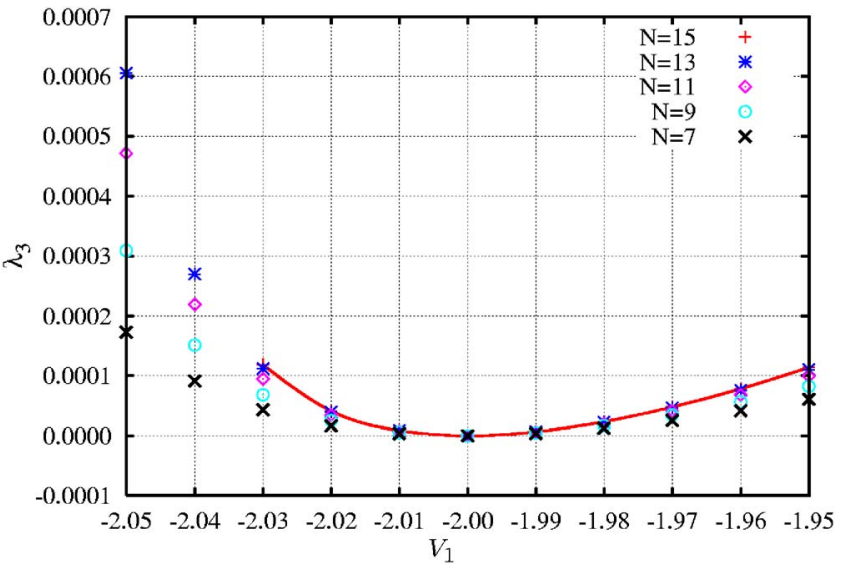

FIG. 4. (Color online) Behavior of the third eigenvalue of the two-site density matrix $\lambda_{3}$ as a function of $V$ in the neighborhood of the phase transition at $V_{1}=-2$ for different sizes at half filling. $\lambda_{3}$ vanishes at $V_{1}=-2$ with very high precision.

pects the interaction to reduce with distance. However, there can be exceptions if the nearest-neighbor interaction is suppressed by the lattice geometry.

The phase diagram of the model represented by the Hamiltonian [Eq. (10)] has been studied as a function of $V_{1}$ and $V_{2}$ by Schmitteckert and Werner. ${ }^{16}$ In this paper, the authors used DMRG to calculate the ground-state curvature. The phase diagram depends on the filling, and commensurability effects are extremely important due to the multiple Umklapp scattering. If we concentrate on half filling and repulsive interactions, we have a charge-density wave (CDW) phase in which the ground state is twofold degenerate with ordering patterns $(\bigcirc \bigcirc \bigcirc)$ and $(\bigcirc \bigcirc)$. Here, $\bigcirc$ denotes a vacant and denotes an occupied site. In phase CDW II, the ground state is fourfold degenerate with order-

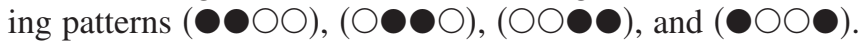
We will follow Ref. 16 and study the critical parameters along the line $V_{2}=5-2 \mathrm{~V}$. For example, studying systems of sizes up to $L=60$, they obtained a critical point for the transition between the CDW I phase and the Luttinger Liquid phase as $\left(V_{1, \mathrm{c}}, V_{2, \mathrm{c}}\right)=(2.4 \pm 0.05,0.2 \pm 0.1)$. In Fig. 5, we show results for the ground-state bond entropy as a function of $V_{2}$ for along the previous mentioned line for $L=36$ and $N=18$. Even from the small size used, we can accurately determine the critical $V_{2}$ as $V_{2, \mathrm{c}}=0.280$, which is within the error bars previously given by Schmitteckert and Werner. ${ }^{16}$ The determination of the critical parameter for the transition between CDW I and the Luttinger liquid is done with much less numerical work as compared to the finite-size analysis of excitation gaps and the ground-state curvate in Ref. 16. Notably, the finite-size corrections are smaller, e.g., the results of the same analysis with $L=18$ already give a critical parameter of $V_{2, \mathrm{c}}=0.277$. In Fig. 6, we show the numerical results for the value of the next-neighbor interaction $V_{2}$ in which we have a local maximum of $S_{\text {bond }}$ (along the same line as before) as a function of the inverse of the total length of the system, $1 / L$. We have calculated numerically the bond entropy at each size with an interval of 0.001 in $V_{2}$ in the region around the maximum of $S_{\text {bond }}$, except in the case of $L=36$ where we have used an interval of $=0.0002$. The val- 


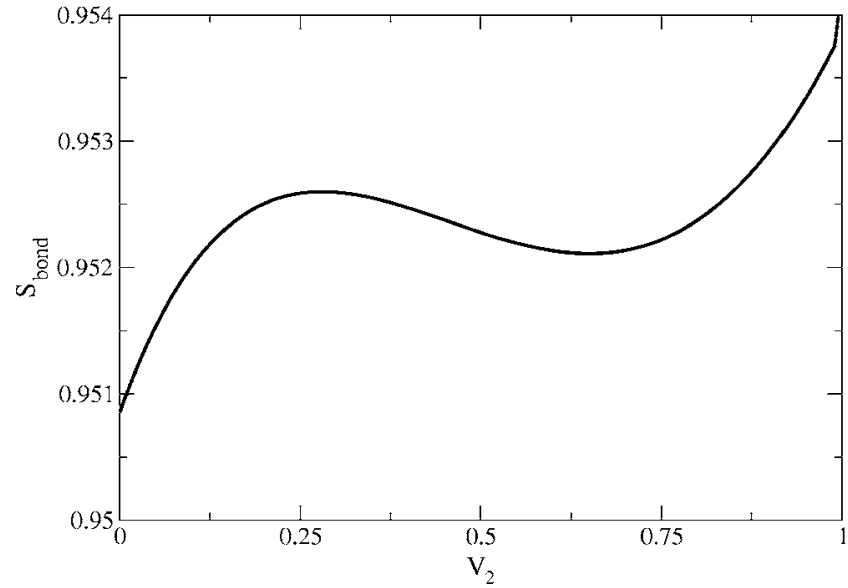

FIG. 5. Bond entropy as a function of $V_{2}$ in the line $V_{2}=5$ $-2 V_{1}$ for $L=36$ and $N=18$. The maximum can be used to estimate the critical point at $V_{2, c}=0.280$ with very high precision.

ues of $V_{2}$ in the maximum were obtained through a secondorder polynomial fit of the numerical results for $S_{\text {bond }}$. The actual value of the interval used was not very critical as the fits were very good. With another second-order polynomial fit, we can extrapolate the calculated values to obtain the result for the thermodynamic limit $V_{2, c}=0.2814 \pm 0.0001$. In the inset of the figure, we can see the numerical results used in the extrapolation of the value of the maximum of the bond entropy in the critical point. The extrapolated value being $S_{\max }=0.95385 \pm 0.00001$.

In this case, the bond entropy and the two-site density matrix do not give very useful information about the phase transitions to charge-density wave phases with ordering patterns with basic sizes bigger than 2 . We obtain no clear signature in the bond entropy for the quantum phase transition to CDW II. One may have to study entropies of density matrices of blocks with at least the size of the basic ordered block of the phases we are looking at. Also, one could try to study the bond entropy for the excited states. The numerical determination of critical parameters in this case is out of the scope of this study of the bond entropy, and will be the subject of future work. We note that in the case of CDW II, the ground state is fourfold degenerate in the thermodynamic limit. However, the degeneracy of the two lowest-lying states is lifted by finite-size effects.

\section{CONCLUSIONS}

The bond entropy defined as the von Neumann entropy of the two-site density matrix can be a very effective tool for the study of phase transitions and critical parameters. Its behavior depends on the correlations in the ground state of the system.

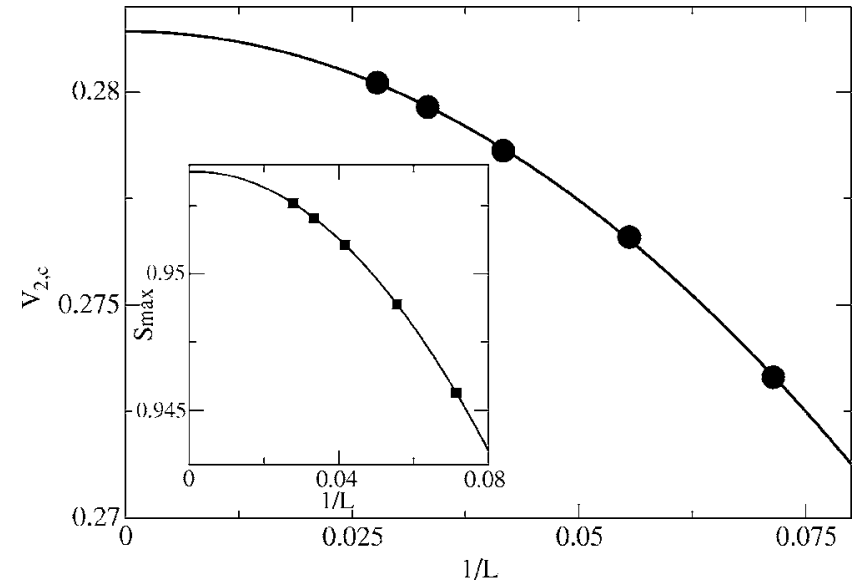

FIG. 6. Finite-size scaling for the position of the maximum of the bond entropy $V_{2, c}$ as a function of $1 / L$. The continuous line is a second-order polynomial fit. It is used to extrapolate the value for $L=\infty$. In the inset, we show the value of the local maximum of $S_{\text {bond }}$ as a function of $1 / L$; a second-order polynomial also fits very well the numerical results.

We have studied the bond entropy for a model of spinless fermions with nearest-neighbor interactions and periodic boundary conditions. The size dependence of its behavior near the two critical points in the model has been studied in detail, showing an amazing precision in the estimation of the critical parameter. We have also studied a model with nextnearest-neighbor interactions. We could determine the critical point of the phase transition from the Luttinger liquid to the CDW I state with an ordering pattern of period 2. If the fundamental block contains only two sites, we show that the bond entropy displays a clear signature of the quantum phase transitions and allows for the determination of the critical parameters. The bond entropy of the ground state could not be used for the transition to CDW II with ordering pattern of period 4. In this case, we may have to turn to a block entropy of higher size. In general, we can say that the bond entropy can be used as a numerical indicator for phase transitions but the actual behavior of the bond entropy is not universal and will depend on the QPT under study. Our results should open the way to the numerical study of phase transitions with small-sized systems.

\section{ACKNOWLEDGMENTS}

R.A.M. wishes to acknowledge useful discussions with J. Dukelsky. He also acknowledges financial support at the Instituto de Estructura de la Materia-CSIC by an I3P contract funded by the European Social Fund. This work is supported in part by the Spanish Government Grant No. FIS200612783-C03-01 and joint CAM-CSIC Grant No. $200650 \mathrm{M} 012$. 
${ }^{1}$ S. Sachdev, Quantum Phase Transitions (Cambridge University Press, Cambridge, 1999).

${ }^{2}$ S. L. Sondhi, S. M. Girvin, J. P. Carini, and D. Shahar, Rev. Mod. Phys. 69, 315 (1997).

${ }^{3}$ F. D. M. Haldane, Phys. Lett. 93A, 464 (1983); F. Verstraete, M. Popp, and J. I. Cirac, Phys. Rev. Lett. 92, 027901 (2004).

${ }^{4}$ F. Verstraete, M. A. Martín-Delgado, and J. I. Cirac, Phys. Rev. Lett. 92, 087201 (2004).

${ }^{5}$ F. Mintert, A. R. R. Carvalho, M. Kuś, and A. Buchleitner, Phys. Rep. 415, 207 (2005).

${ }^{6}$ T. J. Osborne and M. A. Nielsen, Phys. Rev. A 66, 032110 (2002).

${ }^{7}$ A. Osterloh, L. Amico, G. Falci, and R. Fazio, Nature (London) 416, 608 (2002).

${ }^{8}$ G. Vidal, J. I. Latorre, E. Rico, and A. Kitaev, Phys. Rev. Lett. 90, 227902 (2003).

${ }^{9}$ J. Vidal, G. Palacios, and R. Mosseri, Phys. Rev. A 69, 022107 (2004).

${ }^{10}$ J. Vidal, R. Mosseri, and J. Dukelsky, Phys. Rev. A 69, 054101
(2004).

${ }^{11}$ S. Hill and W. K. Wootters, Phys. Rev. Lett. 78, 5022 (1997); W. K. Wootters, ibid. 80, 2245 (1998).

${ }^{12}$ V. E. Korepin, Phys. Rev. Lett. 92, 096402 (2004).

${ }^{13}$ J. P. Keating and F. Mezzadri, Phys. Rev. Lett. 94, 050501 (2005).

${ }^{14}$ Shi-Jian Gu, Guang-Shan Tian, and Hai-Qing Lin, New J. Phys. 8, 61 (2006).

${ }^{15}$ P. Jordan and E. Wigner, Z. Phys. 47, 631 (1928).

${ }^{16}$ P. Schmitteckert and R. Werner, Phys. Rev. B 69, 195115 (2004).

${ }^{17}$ N. Nagaosa, Quantum Field Theory in Strongly Correlated Electronic Systems (Springer-Verlag, Berlin, 1999).

${ }^{18}$ E. Lieb and D. Mattis, J. Math. Phys. 3, 749 (1962).

${ }^{19}$ X. Wang and P. Zanardi, Phys. Lett. A 301, 1 (2002).

${ }^{20}$ S. J. Gu, G. S. Tian, and H. Q. Lin, Phys. Rev. A 71, 052322 (2005).

${ }^{21}$ S. R. White, Phys. Rev. Lett. 69, 2863 (1992). 